\title{
Validation of the Dutch version of the quick mild cognitive impairment screen (Qmci-D)
}

\author{
Steven Bunt ${ }^{1 *}$, Rónán O'Caoimh ${ }^{2,3}$, Wim P. Krijnen', D. William Molloy², Geert Pieter Goodijk', \\ Cees P. van der Schans ${ }^{1,4}$ and Hans J S M Hobbelen ${ }^{1}$
}

\begin{abstract}
Background: Differentiating mild cognitive impairment (MCl) from dementia is important, as treatment options differ. There are few short ( $<5 \mathrm{~min}$ ) but accurate screening tools that discriminate between $\mathrm{MCl}$, normal cognition (NC) and dementia, in the Dutch language. The Quick Mild Cognitive Impairment (Qmci) screen is sensitive and specific in differentiating MCI from NC and mild dementia. Given this, we adapted the Qmci for use in Dutch-language countries and validated the Dutch version, the Qmci-D, against the Dutch translation of the Standardised Mini-Mental State Examination (SMMSE-D).

Method: The Qmci was translated into Dutch with a combined qualitative and quantitative approach. In all, 90 participants were recruited from a hospital geriatric clinic (25 with dementia, 30 with MCl, 35 with NC). The Qmci-D and SMMSE-D were administered sequentially but randomly by the same trained rater, blind to the diagnosis.

Results: The Qmci-D was more sensitive than the SMMSE-D in discriminating MCI from dementia, with a significant difference in the area under the curve $(A \cup C), 0.73$ compared to $0.60(p=0.024)$, respectively, and in discriminating dementia from NC, with an AUC of 0.95 compared to $0.89(p=0.006)$. Both screening instruments discriminated $\mathrm{MCl}$ from NC with an AUC of 0.86 (Qmci-D) and 0.84 (SMMSE-D).

Conclusion: The Qmci-D shows similar,(good) accuracy as the SMMSE-D in separating NC from MCl; greater,(albeit fair), accuracy differentiating MCl from dementia, and significantly greater accuracy in separating dementia from NC. Given its brevity and ease of administration, the Qmci-D seems a useful cognitive screen in a Dutch population. Further study with a suitably powered sample against more sensitive screens is now required.
\end{abstract}

Keywords: Validity, Mild cognitive impairment, Dementia, Quick mild cognitive impairment screen, Screening

\section{Background}

The prevalence of mild cognitive impairment (MCI) [1] and dementia [2] is increasing worldwide. Differentiating MCI from dementia is important, because treatment options differ. In particular, pharmaceutical therapy, indicated for treatment of dementia, is inappropriate and potentially even harmful, in those with MCI [3]. Differentiating MCI from normal cognition (NC) is also important, because people with $\mathrm{MCI}$ are at increased risk of developing dementia, compared to aged-matched individuals in the population [4], and early identification may allow prompt intervention $[5,6]$. Few short

\footnotetext{
*Correspondence: s.bunt@pl.hanze.nl

'Research group Healthy Ageing, Allied Health Care and Nursing, Hanze University of Applied Sciences, Eyssoniusplein 18, P.O. Box 30030, 9700 RM, Groningen, The Netherlands

Full list of author information is available at the end of the article
}

(administration time of approximately $5 \mathrm{~min}$ ) cognitive screening instruments are useful in discriminating between $\mathrm{MCI}$ and $\mathrm{NC}$ or dementia. Most are limited by their sensitivity and specificity [7]. Likewise, few are available in the Dutch language. One of the most widely used tools is the Mini-Mental State Examination (MMSE) [8]. The MMSE was standardized to improve reliability, producing the Standardised Mini-Mental State Examination (SMMSE) [9, 10], which is available in a wide variety of languages including Dutch [11]. However, there is limited evidence that either the MMSE or SMMSE are sufficiently accurate in identifying MCI [12], particularly in those with high educational attainment [13].

To address these challenges, the Quick Mild Cognitive Impairment $(\mathrm{Q} m c i)$ screen was developed. Based upon 
the ABCS 135 [14], it was modified to increase its sensitivity to differentiate $\mathrm{NC}$ from $\mathrm{MCI}$, by the addition of logical memory. The Qmci is more sensitive in differentiating MCI from NC than the SMMSE and ABCS 135 [15]. The Qmci has six subtests, orientation, registration, clock drawing, delayed recall, verbal fluency and logical memory. It is scored out of 100 points, can be administered and scored in less than $5 \mathrm{~min}$ and has excellent test-retest reliability [16]. The Qmci correlates highly with the Standardised ADAS-cog, Clinical Dementia Rating scale and the Lawton-Brody activities of daily living scale [17].

The goal of the present study was to adapt the Qmci for use in Dutch-language countries, to validate the Dutch version of the Qmci (Qmci-D) and to compare its sensitivity and specificity in differentiating MCI from $\mathrm{NC}$ and dementia to the most widely used short cognitive screen in the Netherlands, the Dutch version of the SMMSE (SMMSE-D).

\section{Methods \\ Translation}

The translation of the Qmci was performed with a combined qualitative and quantitative approach [18]. The original version of the Qmci was translated to Dutch by a health professional with a good understanding of English, whose primary language is Dutch. This Dutch version was reviewed by an expert panel of Dutch health professionals and researchers and a completed version of the Qmci-D was generated. A professional, native English language-speaking translator, without knowledge of the concepts behind the screening tool, performed the back-translation. The back-translation was then reviewed by the original developers of the Qmci screen, who approved the final version. The $\mathrm{Qmci}$-D was pre-tested on participants with normal cognition before it was used in this study.

\section{Participants}

Consecutive patients were recruited during a four monthperiod, between November 2013 and March 2014, from a geriatric outpatient department in a regional hospital, in the North of the Netherlands, where they were referred for the assessment of cognitive problems. Normal controls were recruited by convenience sampling among healthy participants without cognitive problems, who were accompanying the patients. A diagnosis of dementia (Alzheimer's disease, vascular or mixed dementia subtypes) was made using DSM-IV [19] and NINCDS-ADRDA [20] criteria. A diagnosis of amnestic type MCI was made in patients with objective memory loss, greater than expected with ageing but without loss of social or occupational function, according to the National Institute on
Aging- Alzheimer's Association workgroups diagnostic guidelines for Alzheimer's disease [21]. Participants were excluded if they were younger than 55, if they had active depression (Geriatric Depression Scale $>5$ ), if they weren't able to communicate in Dutch or if they were diagnosed with other MCI or dementia subtypes, including frontotemporal dementia, Parkinson's disease or Lewy Body dementia. Those with frontotemporal, Parkinson's disease and Lewy body dementia were excluded as they present infrequently [22] and typically present with exaggerated functional deficits and different MCI syndromes [23-25].

The MCI and dementia groups underwent the same comprehensive review at baseline including neuropsychological assessment and Magnetic Resonance Imaging or Computerized Tomogram. The purpose and procedure of the research were explained in advance and all participants signed an informed consent before participation in the study. The Medical Ethical Committee of the University Medical Center Groningen evaluated the study and judged that it did not need ethical approval under Dutch law.

A power calculation was performed a priori, to establish the sample size, using the WINPEPI software programme PAIRSetc $[26,27]$. Based upon the original validation results of the Qmci compared to the SMMSE [15], it was expected that the accuracy, as indicated by the area under the curve (AUC) of receiver operating characteristic (ROC) curves, of the Qmci would be $85 \%$ compared to approximately $65 \%$ for the SMMSE, to differentiate MCI from NC. To detect a $20 \%$ (medium to large effect size) difference in sensitivity and specificity, between the two tests, at a significance level of 0.05 and power of $80 \%$, 76-paired observations were required. The sample size needed to distinguish MCI from dementia was not estimated as a significant difference between the Qmci and the SMMSE would not be expected [15].

\section{Data collection}

Demographic data (age and gender) were collected during each visit to the geriatric department. Patients were classified by a consultant geriatrician and divided into three groups (NC, MCI or dementia). A trained rater administered both the Qmci-D (score range 0-100, impaired to normal) and SMMSE-D (score range 0-30, impaired to normal) on the same day, in a counterbalanced fashion, blind to the final diagnosis.

\section{Statistical analysis}

Data were analyzed using SPSS version 20.0 and the statistical programming language $\mathrm{R}$. The Shapiro-Wilk test was used to test for normality. Differences in Qmci-D and SMMSE-D scores, between groups, were tested by 
one-way analysis of variance (ANOVA). Analysis of covariance (ANCOVA) was used to test differences between participant groups, controlling for participant characteristics such as age. Post hoc pair-wise comparisons were performed using the Tukey's honest significant difference (HSD) test. A p-value $<0.05$ was regarded as significant. Bootstrapped ROC curves were generated [28] to analyze the discriminatory characteristics of the Qmci-D and SMMSE-D [29]. Differences between AUCs were calculated using the DeLong approach [30].

\section{Results}

In total, 90 participants, 41 males (46\%) and 49 females (54\%), were included in this study. In this sample, 35 (39 \%) had NC, 30 (33\%) had MCI and 25 (28 \%) were diagnosed with dementia. The overall mean age of the sample was 72.9, standard deviation (SD) of 9.1 years. The NC group (mean age 68.7) was younger than the MCI (mean age 79.1) and dementia (mean age 79.2) groups $(p<0.001)$. The NC group had a mean Qmci-D score of 64 (SD 10.5) and a mean SMMSE-D score of 28 (SD 1.8). The mean $\mathrm{Qmci- \textrm {D }}$ score for the $\mathrm{MCI}$ group was 46 (SD 11.8) compared with 24 (SD 2.9) for the SMMSE-D, while the dementia group scored 34 (SD 15.8) for the Qmci-D and 22 (SD 4.4) for the SMMSE-D. These scores and demographic data are summarized in Table 1.

One-way ANCOVA, used to test for differences in SMMSE-D scores between the three groups (NC, $\mathrm{MCI}$ and dementia), showed that the mean scores differed significantly across the three groups $(F=20.55$, $\mathrm{df}=3,86, p<0.001)$. Post hoc pair-wise comparisons using the Tukey's HSD test showed significant mean differences between groups $(p<0.05)$. The mean scores for the Qmci-D also differed significantly between the three groups; $(F=33.24, \mathrm{df}=3,86, p<0.001)$. The differences between groups are presented as boxplots in Fig. 1. ANOVA post-hoc testing for multiple comparisons, showed a significant difference in mean scores between the dementia and NC groups, for both tests (see Table 2).

Comparisons of the AUC of ROC curves and the point estimates (cut-off scores), providing the optimal sensitivity and specificity, are presented in Fig. 2. These show that the Qmci-D was more accurate than the SMMSE-D in discriminating between dementia and NC, with an AUC of 0.95 (95\% CI 0.90-0.99), compared to 0.89 (95 \% CI $0.80-0.96)$ for the SMMSE-D (see Fig. 2a, b). The difference was significant $(p=0.006)$. Both the Qmci-D and the SMMSE-D discriminated MCI from NC, with ANOVA post-hoc tests showing a significant mean difference between the MCI and NC groups $(p<0.001)$. The AUC of the Qmci-D, in discriminating $\mathrm{MCI}$ from $\mathrm{NC}$, was marginally greater at 0.86 (95\% CI $0.77-0.95)$, compared to 0.84 (95 \% CI 0.74-0.94) for the SMMSE-D (see Fig. 2c, d). This difference was non-significant $(p=0.335)$. At the point estimate the Qmci-D had a sensitivity of $70 \%$ and specificity of $94 \%$ compared to a sensitivity of only $60 \%$ and a similar specificity of $94 \%$ for the SMMSE-D. As for the discrimination of MCI from dementia, ANOVA posthoc tests, showed a significant mean difference $(p<0.001)$ between these participants with the Qmci-D. The difference between scores for the MCI and dementia groups, for the SMMSE-D $(p=1.02)$ was not significant. The score test for homogeneity of variances across groups in ANCOVA indicated that homogeneity for the Qmci is rejected. Heteroscedasticity-corrected SEs and Tests after ANCOVA [31] indicated that the difference in Qmci-D mean scores after correction for age, between MCI and

Table 1 Characteristics of patients with mild cognitive impairment (MCl), dementia, and participants with normal cognition (NC) including their Quick Mild Cognitive Impairment (Qmci-D) screen and Standardised Mini-Mental State Examination (SMMSE-D) scores

\begin{tabular}{|c|c|c|c|}
\hline Group & Dementia & $\mathrm{MCl}$ & Normal cognition \\
\hline Number of participants & 25 & 30 & 35 \\
\hline \multicolumn{4}{|l|}{ Age } \\
\hline Mean (SD) & $79.2(5.7)$ & $79.1(5.9)$ & $68.7(9.0)$ \\
\hline Median (IQR) & $80(84-76=8)$ & $80(83-74=9)$ & $68(77-61=16)$ \\
\hline \multicolumn{4}{|l|}{ Gender } \\
\hline (\% female) & $48 \%$ & $56 \%$ & $57 \%$ \\
\hline \multicolumn{4}{|l|}{ Qmci-D (range 0-100) } \\
\hline Mean Score (SD) & $34(15.8)$ & $46(11.8)$ & $64(10.5)$ \\
\hline Median Score (IQR) & $35.5(48-21=17)$ & $46.8(54-38=16)$ & $64.5(72-55=17)$ \\
\hline \multicolumn{4}{|l|}{ SMMSE-D (range 0-30) } \\
\hline Mean Score (SD) & $22(4.4)$ & $24(2.9)$ & $28(1.8)$ \\
\hline Median Score (IQR) & $23(26-18=8)$ & $23.5(26-22=4)$ & $28(29-27=2)$ \\
\hline
\end{tabular}

SD Standard Deviation, IQR inter-quartile range, IQR Q1-Q3, Q1 1st Quartile, Q3 3rd quartile 

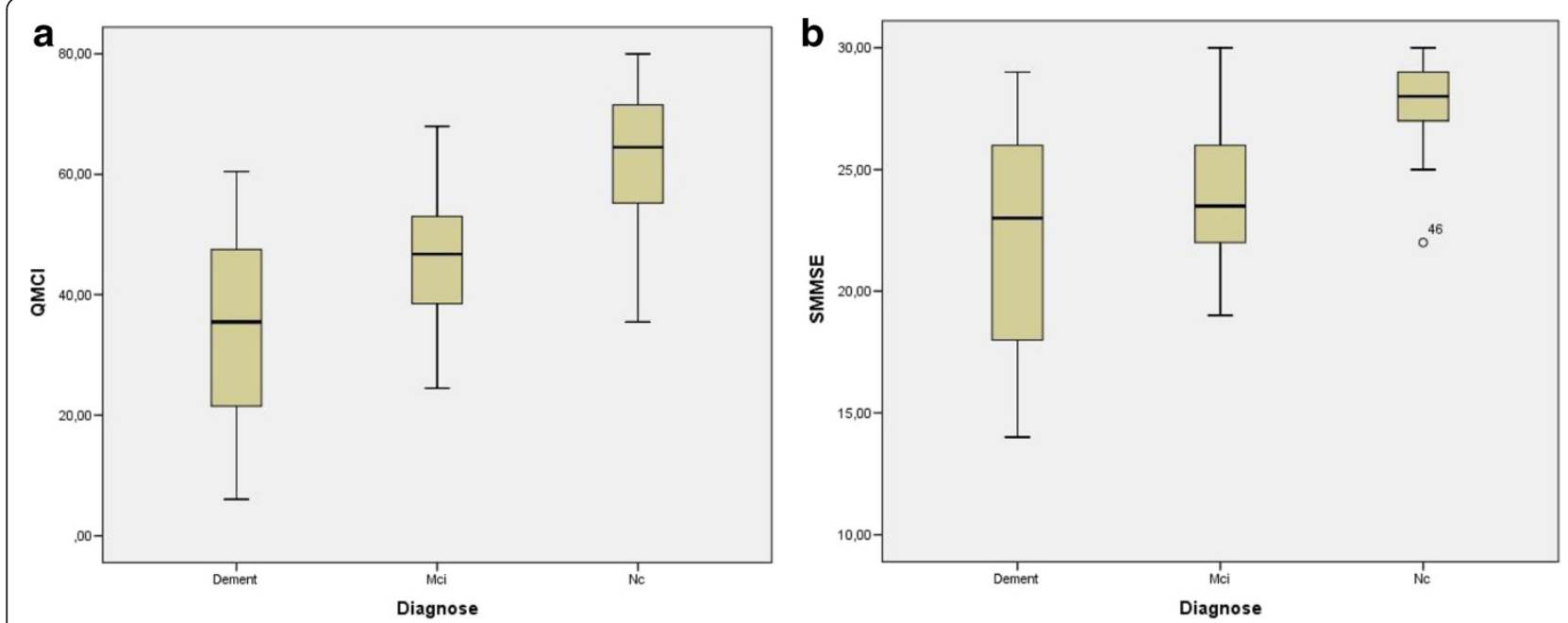

Fig. 1 Boxplots representing scores on the (a) Qmci-D (score range 0-100) and (b) SMMSE-D (score range 0-30) in dementia, MCI and normal cognition groups

$\mathrm{NC}$ as well as between MCI and dementia, were significantly different from zero. The ability of the Qmci-D, to discriminate $\mathrm{MCI}$ from dementia, was significantly greater $(p=0.024)$ than the SMMSE-D, AUC of 0.73 (95\% CI 0.59-0.85) compared to 0.60 (95\% CI 0.45-0.75), respectively (See Fig. 2e, f). At the point estimate the Qmci-D had a modest sensitivity of $64 \%$ to differentiate MCI from dementia, although this compared favorably to the SMMSE-D with a sensitivity of only $28 \%$.

When moderate and severe dementia cases were removed from analysis, the AUC of the Qmci-D and SMMSE-D for differentiating MCI from mild dementia cases alone was unchanged at 0.62 and $0.54, p=0.03$, respectively.

\section{Discussion}

The goals of this study were to adapt the Qmci for use in Dutch-language countries and to explore its concurrent validity against the most commonly used short cognitive screen in Dutch, the SMMSE-D. The results show that the Qmci-D is more accurate than the SMMSE-D in differentiating dementia from NC. It had only fair accuracy (AUC 0.73) at differentiating MCI from dementia, although it was significantly more accurate than the SMMSE (AUC 0.60). In this study however, the SMMSE-D wasn't able to discriminate MCI from dementia with a very poor sensitivity of $24 \%$, particularly when moderate to severe cases were excluded. Based upon this data it would appear that both instruments have limited ability to separate MCI from dementia. This is markedly different from the initial validation of the English language version of the Qmci against the SMMSE [15], which in a much larger sample of almost 1000 Canadians, suggested that both had excellent accuracy (AUC >0.90), although it showed no significant difference between the two instruments in their ability to distinguish MCI from dementia. There was also no significant difference between the tests' ability to discriminate between MCI and NC in this sample, although the accuracy of both tests was good, suggesting that both were able to separate MCI from NC. This again differs from the initial validation, where the Qmci showed

Table 2 ANOVA Post-Hoc tests: multiple comparisons between NC, MCl and Dementia groups

\begin{tabular}{|c|c|c|c|c|c|c|c|}
\hline \multirow{2}{*}{$\begin{array}{l}\text { Dependent } \\
\text { Variable }\end{array}$} & \multirow[t]{2}{*}{ Group } & \multirow[t]{2}{*}{ Group } & \multirow{2}{*}{$\begin{array}{l}\text { Mean } \\
\text { Difference }\end{array}$} & \multirow{2}{*}{$\begin{array}{l}\text { Std. } \\
\text { Error }\end{array}$} & \multirow[t]{2}{*}{$P$-value } & \multicolumn{2}{|c|}{$95 \%$ Confidence Interval } \\
\hline & & & & & & Lower Bound & Upper Bound \\
\hline \multirow[t]{3}{*}{ Qmci-D } & $\mathrm{MCl}$ & Dementia & 12.56 & 3.40 & 0.001 & 4.25 & 20.87 \\
\hline & NC & $\mathrm{MCl}$ & 17.19 & 3.13 & $<0.001$ & 9.55 & 24.82 \\
\hline & NC & Dementia & 29.75 & 3.29 & $<0.001$ & 21.71 & 37.78 \\
\hline \multirow[t]{3}{*}{ SMMSE-D } & $\mathrm{MCl}$ & Dementia & 1.79 & 0.83 & 0.102 & -0.24 & 3.83 \\
\hline & NC & $\mathrm{MCl}$ & $3.70^{*}$ & 0.77 & $<0.001$ & 1.84 & 5.58 \\
\hline & NC & Dementia & $5.50^{*}$ & 0.81 & $<0.001$ & 3.54 & 7.47 \\
\hline
\end{tabular}



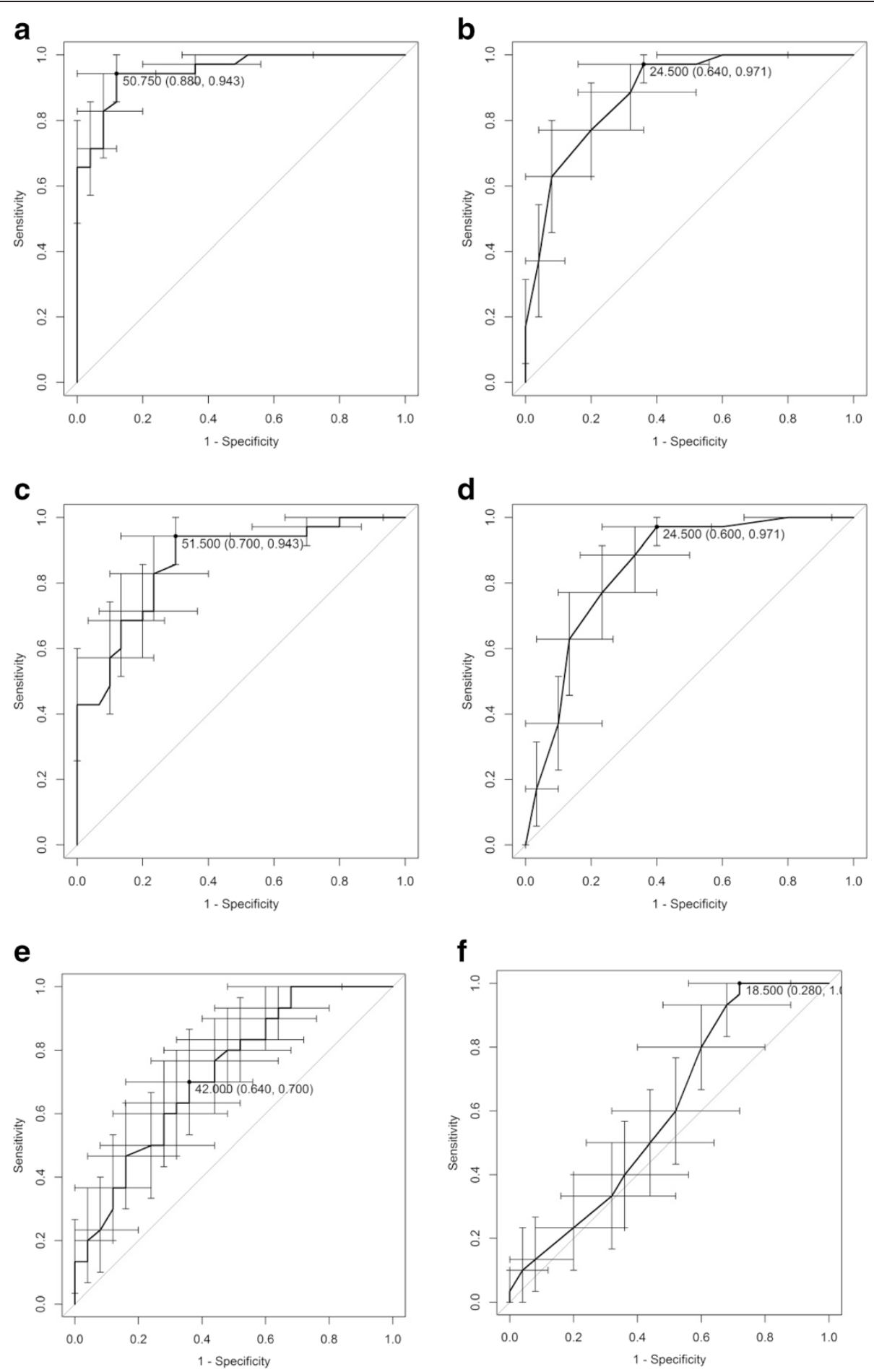

Fig. 2 Bootstrapped ROC curves with $95 \%$ confidence intervals demonstrating sensitivities and specificities of (a) Qmci-D and (b) SMMSE-D in differentiating dementia from normal cognition, the (c) Qmci-D and (d) SMMSE-D in differentiating MCl from normal cognition, and the (e) Qmci-D and (f) SMMSE-D in differentiating MCI from dementia

significantly greater accuracy over the SMMSE. This discrepancy may relate to the small sample size, suggesting that this study was underpowered to show superiority of one instrument over the other. This said, the goal of this study was not to show superiority of a Dutch language version of the $\mathrm{Q} m c i$, the $\mathrm{Q} m c i-\mathrm{D}$, rather it was to show the concurrent validity of the translation against a widely used screening instrument. 
The strength of this study is the robust analysis with bootstrapped ROC curves and $95 \%$ confidence intervals, to identify the discriminatory characteristics of both screening tools. This method provides more accurate results than non-bootstrapped methods, especially when analyzing smaller sample sizes. The $95 \%$ confidence intervals obtained from the bootstrap and the asymptotic approach [30], were in all cases virtually equal. This indicates that the intervals are valid.

The study has limitations. First, the diagnosis of MCI was based on clinical criteria, which may have increased the heterogeneity of this group and led to some bias. However, no single gold standard criterion for MCI exists and there is still a lack of uniformity in the clinical diagnosis of MCI between studies [32]. This said, in this study an objective history of cognitive decline over time was obtained from each patient's collateral (family member or caregiver) and assessed by neuropsychological testing, independent of the results of the short cognitive screens, in keeping with the National Institute on Aging Alzheimer's Association diagnostic guidelines [21]. Second, the NC group consisted of participants recruited by convenience sampling from healthy relatives or caregivers attending with patients. These participants were significantly younger than patients with MCI or dementia. This could have increased heterogeneity and created bias, explaining why there was no significant difference in the ability of both instruments to distinguish $\mathrm{MCI}$ from $\mathrm{NC}$, unlike that seen in the initial validation of the Qmci [15]. Patients with MCI and dementia were, however, well matched for age and gender. ANCOVA testing and post-hoc analysis confirmed that differences in mean test score were not attributable to age. Furthermore, the educational status of patients was not recorded routinely, which may also have created some bias. Third, the sample size was small and did not exceed the desired 76-paired observations, calculated as the sample size to detect differences in accuracy between participants with $\mathrm{MCI}$ and NC for the screening tools. Fourth, the study excluded those with active depression and less prevalent dementia subtypes as described above. Active depression was excluded as these patients may have slower reaction times and processing speeds [33]. Frontotemporal, Parkinson's disease and Lewy body dementia often present with exaggerated functional deficits potentially clouding the diagnosis of $\mathrm{MCI}$, the focus of this study. This may have caused the sample to be less representative and created some spectrum bias, limiting the generalizability of the results. Finally, the study compared the Qmci-D only with the SMMSED. This was because the SMMSE is the most widely used short cognitive screen [34] and in the initial validation of the English language version of the Qmci the comparator was the SMMSE, allowing direct comparison with the results of that study [15]. The authors acknowledge the importance of future validation against other short, albeit longer, screens including the Dutch version of the Montreal cognitive assessment (MoCA) [35, 36], the Addenbrooke's Cognitive Examination-Revised [37] and shorter instruments like the Mini-Addenbrooke's Cognitive Examination (M-ACE) [38]. The authors also caution that screening for cognitive impairment continues to have challenges and in clinical practice [39] it remains only one part of a comprehensive assessment of cognition, and should not be relied upon exclusively.

\section{Conclusion}

In conclusion, this study shows the concurrent validity of the Qmci-D against the SMMSE-D. The data suggests that the Qmci-D, although statistically significantly more accurate than the SMMSE-D, is limited in its ability to differentiate MCI from dementia. The results also suggest that the accuracy of both instruments at distinguishing MCI from NC was good although the Qmci was more accurate than the SMMSE in separating dementia from NC in a Dutch speaking population. Given this, albeit limited analysis in a small sample, as well as its brevity and ease of administration $[15-17,40]$, the Dutch version of the $\mathrm{Q} m c i$, the $\mathrm{Q} m c i-\mathrm{D}$, appears useful as a short cognitive screen. Further research is now required to confirm these findings with a larger sample including other dementia subtypes and to compare the test to other cognitive screens including the MoCA and M-ACE.

\section{Abbreviations}

ABCS 135: AB Cognitive Screen; ADAS-cog: Alzheimer's Disease Assessment Scale - cognition; ANCOVA: Analysis of covariance; ANOVA: Analysis of variance; AUC: Area under the curve; DSM-IV: Diagnostic and Statistical Manual of Mental Disorders-IV; IQR: Inter-quartile range; M-ACE: MiniAddenbrook's Cognitive Examination; MCl: Mild cognitive impairment; MMSE: Mini-Mental State Examination; MoCA: Montreal cognitive assessment; NC: Normal cognition; NINCDS-ADRDA: National Institute of Neurological and Communicative Disorders and Stroke and the Alzheimer's Disease and Related Disorders Association; SMMSE-D: Dutch translation of the Standardised Mini-Mental State Examination; Qmci: Quick mild cognitive impairment screen; ROC: Receiver operating characteristic; SD: Standard deviation.

\section{Competing interests}

The author(s) declare that they have no competing interests.

\section{Authors' contributions}

SB planned the study, carried out the project management of this study, and led the writing process of the paper. $\mathrm{HH}$ and $\mathrm{CS}$ took part in setting up the research design, supervised the project, and were part of the expert panel during the translation process. WK helped analyzing the results and carrying out the statistical analysis. GPG assisted in carrying out the research project at the Nij Smellinghe Hospital in Drachten (Netherlands) and had a significant role in gathering data. DM and ROC took part in setting up the design for this study and 
participated in the back-translation process.

All authors had input in, and approved of the final manuscript.

\section{Acknowledgements}

The authors like to thank the geriatric department of the Nij Smellinghe Hospital in Drachten (The Netherlands) for their willingness to participate in this study and their referrals of patients to this study.

\section{Author details}

${ }^{1}$ Research group Healthy Ageing, Allied Health Care and Nursing, Hanze University of Applied Sciences, Eyssoniusplein 18, P.O. Box 30030, 9700 RM, Groningen, The Netherlands. ${ }^{2}$ Centre for Gerontology and Rehabilitation, University College Cork, St Finbarrs Hospital, Douglas Road, Cork City, Ireland. ${ }^{3}$ Health Research Board, Clinical Research Facility Galway, National University of Ireland, Galway, Ireland. ${ }^{4}$ Department of Rehabilitation, University Medical Center Groningen, University of Groningen, Groningen, The Netherlands.

\section{Received: 19 May 2015 Accepted: 28 September 2015 Published online: 02 October 2015}

\section{References}

1. Ward A, Arrighi HM, Michels S, Cedarbaum JM. Mild cognitive impairment: disparity of incidence and prevalence estimates. Alzheimers Dement. 2012;8(1):14-21.

2. Prince $M$, Bryce $R$, Albanese E, Wimo A, Ribeiro W, Ferri CP. The global prevalence of dementia: a systematic review and metaanalysis. Alzheimers Dement. 2013;9(1):63-75. e2.

3. Tricco AC, Soobiah C, Berliner S, Ho JM, Ng CH, Ashoor HM, et al. Efficacy and safety of cognitive enhancers for patients with mild cognitive impairment: a systematic review and meta-analysis. CMAJ. 2013;185(16):1393-401

4. Mitchell AJ, Shiri-Feshki M. Rate of progression of mild cognitive impairment to dementia-meta-analysis of 41 robust inception cohort studies. Acta Psychiatr Scand. 2009;119(4):252-65.

5. Fiatarone Singh MA, Gates N, Saigal N, Wilson GC, Meiklejohn J, Brodaty H, et al. The Study of Mental and Resistance Training (SMART) study-resistance training and/or cognitive training in mild cognitive impairment: a randomized, double-blind, double-sham controlled trial. J Am Med Dir Assoc. 2014;15(12):873-80.

6. O'Caoimh R, Sato S, Wall J, Igras E, Foley MJ, Timmons S, et al. Potential for a "Memory Gym" Intervention to Delay Conversion of Mild Cognitive Impairment to Dementia. Journal of the American Medical Directors Association. 2015, Feb 21. pii: S1525-8610(15)00082-1. doi:10.1016/ j.jamda.2015.01.081.

7. Ritchie K, Artero S, Touchon J. Classification criteria for mild cognitive impairment: a population-based validation study. Neurology. 2001;56(1):37-42.

8. Folstein MF, Folstein SE, McHugh PR. "Mini-mental state". A practical method for grading the cognitive state of patients for the clinician. J Psychiatr Res. 1975;12(3):189-98.

9. Molloy DW, Alemayehu E, Roberts R. Reliability of a standardized minimental state examination compared with the traditional mini-mental state examination. Am J Psychiatry. 1991;148(1):102-5.

10. Molloy DW, Standish TI. A guide to the standardized mini-mental state examination. Int Psychogeriatr. 1997;9 Suppl 1:87-94. discussion 143-50.

11. Kok, R.M., Verheij, F.R.J. Gestandaardiseerde versie van de Mini-Mental State Examination. 2002.

12. Mitchell AJ. A meta-analysis of the accuracy of the mini-mental state examination in the detection of dementia and mild cognitive impairment. J Psychiatr Res. 2009;43(4):411-31.

13. Crum RM, Anthony JC, Bassett SS, Folstein MF. Population-based norms for the mini-mental state examination by age and educational level. JAMA. 1993;269(18):2386-91.

14. Molloy DW, Standish TI, Lewis DL. Screening for mild cognitive impairment: comparing the SMMSE and the ABCS. Can J Psychiatry. 2005;50(1):52-8.

15. O'Caoimh R, Gao Y, McGlade C, Healy L, Gallagher P, Timmons S, et al. Comparison of the quick mild cognitive impairment (Qmci) screen and the SMMSE in screening for mild cognitive impairment. Age Ageing. 2012;41(5):624-9.

16. O'Caoimh R, Gao Y, Gallagher PF, Eustace J, McGlade C, Molloy DW. Which part of the quick mild cognitive impairment screen (Qmci) discriminates between normal cognition, mild cognitive impairment and dementia? Age Ageing. 2013;42(3):324-30.

17. O'Caoimh R, Svendrovski A, Johnston BC, Gao Y, McGlade C, Eustace J, et al. The quick mild cognitive impairment screen correlated with the standardized alzheimer's disease assessment scale-cognitive section in clinical trials. J Clin Epidemiol. 2014;67(1):87-92.

18. Beaton DE, Bombardier C, Guillemin F, Ferraz MB. Guidelines for the process of cross-cultural adaptation of self-report measures. Spine (Phila Pa 1976). 2000;25(24):3186-91.

19. American Psychiatric Association. Diagnostic and Statistical Manual of Mental Disorders. 4th ed. Washington, DC: American Psychiatric Association; 1994.

20. McKhann G, Drachman D, Folstein M, Katzman R, Price D, Stadlan EM. Clinical diagnosis of Alzheimer's disease: report of the NINCDS-ADRDA Work Group under the auspices of Department of Health and Human Services Task Force on Alzheimer's Disease. Neurology. 1984;34(7):939-44.

21. Albert MS, DeKosky ST, Dickson D, Dubois B, Feldman HH, Fox NC, et al. The diagnosis of mild cognitive impairment due to Alzheimer's disease: recommendations from the National Institute on Aging-Alzheimer's Association workgroups on diagnostic guidelines for Alzheimer's disease. Alzheimers Dement. 2011;7(3):270-9.

22. Brunnstrom H, Gustafson L, Passant U, Englund E. Prevalence of dementia subtypes: a 30-year retrospective survey of neuropathological reports. Arch Gerontol Geriatr. 2009;49(1):146-9.

23. de Mendonca A, Ribeiro F, Guerreiro M, Garcia C. Frontotemporal mild cognitive impairment. J Alzheimers Dis. 2004;6(1):1-9.

24. Caviness JN, Driver-Dunckley E, Connor DJ, Sabbagh MN, Hentz JG, Noble B, et al. Defining mild cognitive impairment in Parkinson's disease. Mov Disord. 2007;22(9):1272-7.

25. Yoon JH, Lee JE, Yong SW, Moon SY, Lee PH. The mild cognitive impairment stage of dementia with Lewy bodies and Parkinson disease: a comparison of cognitive profiles. Alzheimer Dis Assoc Disord. 2014;28(2):151-5.

26. Gahlinger PMAJ. Computer programs for epidemiologic analysis: PEPI. 2nd ed. Stone Mountain: USD Inc.; 1995.

27. Abramson JH. WINPEPI updated: computer programs for epidemiologists, and their teaching potential. Epidemiol Perspect Innov. 2011;8(1):1. -5573-8-1.

28. Robin X, Turck N, Hainard A, Tiberti N, Lisacek F, Sanchez JC, et al. pROC: an open-source package for $\mathrm{R}$ and $\mathrm{S}+$ to analyze and compare ROC curves. BMC Bioinf. 2011;12:77. -2105-12-77.

29. Carpenter J, Bithell J. Bootstrap confidence intervals: when, which, what? A practical guide for medical statisticians. Stat Med. 2000;19(9):1141-64.

30. DeLong E, DeLong D, Clarke-Pearson D. Comparing the areas under Two or more correlated receiver operating characteristic curves: A nonparametric approach. Biometrics. 1988;44(3):837-45

31. White $\mathrm{H}$. A heteroskedastic consistent covariance matric estimator and a direct test of heteroskedasticity. Econometrica. 1980;48:817-38.

32. Christa Maree Stephan B, Minett T, Pagett E, Siervo M, Brayne C, McKeith IG. Diagnosing Mild Cognitive Impairment (MCI) in clinical trials: a systematic review. BMJ Open. 2013;3(2):e001909. doi:10.1136/bmjopen-2012-001909. Print 2013.

33. Iverson GL. Sensitivity of computerized neuropsychological screening in depressed university students. Clin Neuropsychol. 2006;20(4):695-701.

34. Ismail Z, Rajji TK, Shulman Kl. Brief cognitive screening instruments: an update. Int J Geriatr Psychiatry. 2010;25(2):111-20.

35. Nasreddine ZS, Phillips NA, Bedirian V, Charbonneau S, Whitehead V, Collin I, et al. The Montreal Cognitive Assessment, MoCA: a brief screening tool for mild cognitive impairment. J Am Geriatr Soc. 2005;53(4):695-9.

36. Thissen AJ, van Bergen F, de Jonghe JF, Kessels RP, Dautzenberg PL. Applicability and validity of the Dutch version of the Montreal Cognitive Assessment (moCA-d) in diagnosing MCl. Tijdschr Gerontol Geriatr. 2010;41(6):231-40

37. Larner AJ, Mitchell AJ. A meta-analysis of the accuracy of the Addenbrooke's Cognitive Examination (ACE) and the Addenbrooke's Cognitive Examination-Revised (ACE-R) in the detection of dementia. Int Psychogeriatr. 2014;26(4):555-63.

38. Hsieh S, McGrory S, Leslie F, Dawson K, Ahmed S, Butler CR, et al. The MiniAddenbrooke's Cognitive Examination: a new assessment tool for dementia. Dement Geriatr Cogn Disord. 2015;39(1-2):1-11. 
39. Lin JS, O'Connor E, Rossom RC, Perdue LA, Eckstrom E. Screening for cognitive impairment in older adults: A systematic review for the U.S. Preventive Services Task Force. Ann Intern Med. 2013;159(9):601-12.

40. O'Caoimh R, Timmons S, Molloy DW. Comparison of the Quick Mild

Cognitive Impairment Screen (Qmci) to the Montreal Cognitive Assessment. Ir J Med Sci 2013;182S(6):286.

Submit your next manuscript to BioMed Central and take full advantage of:

- Convenient online submission

- Thorough peer review

- No space constraints or color figure charges

- Immediate publication on acceptance

- Inclusion in PubMed, CAS, Scopus and Google Scholar

- Research which is freely available for redistribution 